\title{
On the Combined Effects of a Magnetic Field and Rotation on Acoustic Modes
}

\author{
L. Bigot
}

Observatoire de la Côte d'Azur, Nice, France; e-mail: lbigot@obs-nice.fr

W. A. Dziembowski

N. Copernicus Astronomical center, Warsaw, Poland

\begin{abstract}
We present new results on the effects of a strong dipole magnetic field and rotation on p-mode oscillations. We take into account both Coriolis and centrifugal distortion as manifestations of rotation. We apply these improvements to the case of dipole $(\ell=1)$ modes. We show that these modes, during the pulsation cycle, move the stellar surface in a plane, which is inclined from both magnetic and rotation axes. In general, the displacement vector describes an ellipse in that plane. This work is devoted to the study of the roAp star pulsations.
\end{abstract}

\section{Introduction}

The rapidly oscillating Ap (roAp) stars are variable main sequence stars showing rapid light variations of a few minutes associated to acoustic modes. The amplitudes of these oscillations are about $1 \mathrm{mmag}$. To date, 32 candidates have been identified since their discovery two decades ago by Kurtz (1982). The particularity of these stars is that they show very strong global magnetic fields of kilogauss strengths. This huge field deeply affects the properties of oscillations in the star. The most observed modes in roAp stars are the dipole modes.

\section{Magnetic and rotation effects on acoustic modes}

In order to calculate the effects of a magnetic field we assume for simplicity a dipole field. The Lorentz force strongly affects p-mode propagation in the outer layers of the star where the magnetic pressure is much larger than the gas pressure. In this part of the star Dziembowski \& Goode (1996), Bigot et al. (2000) or Cunha \& Gough (2000) solved MHD equations with plan-parallel approximation. The results show important shifts of frequencies of several $\mu \mathrm{Hz}$, due to the fact that the field modifies the speed of the wave propagation in the resonant cavity. An important effect of the magnetic field is that it partially removes the degeneracy of frequencies in $m$ (azimuthal order) since it breaks the spherical symmetry of the problem.

Because of the inclination between magnetic and rotation axes, the effect of rotation is to couple, for each degree $\ell$, the $(2 \ell+1)$ eigenvectors corresponding 
to the different values of $m$, so that the general displacement vector is a linear combination of these $(2 \ell+1)$ coupled harmonics,

$$
\vec{\xi}=\sum_{m=-\ell}^{\ell} \alpha_{m} \vec{\xi}_{m}
$$

In this formalism it can be shown (e.g. Dziembowki \& Goode, 1985) that the linearized equation for adiabatic oscillations leads to an eigenvalue system

$$
\sum_{m=-\ell}^{\ell} \alpha_{m}\left\{O_{j m}-\omega^{2} \delta_{j m}\right\}=0 \quad j=-\ell, \ldots, \ell
$$

with $O_{j m}$ a matrix which corresponds to the projection of the Lorentz, Coriolis and centrifugal operators onto the $\vec{\xi}_{m}$ (see Bigot \& Dziembowski, 2001). The $(2 \ell+1)$ eigenvalues are the frequencies $\omega$ of the modes. The corresponding eigenvectors are the coefficients of the coupling, $\alpha_{m}$. Both Coriolis and centrifugal forces have to be taken into account in the problem, see Dziembowski \& Goode (1985), Bigot \& Dziembowski (2001). The latter is the dominant contribution of the rotation shift of frequency in roAp stars, mainly due to the fact that they oscillate with large overtones.

\section{Application to dipole modes}

Dipole modes are of special importance in roAp pulsations since they are the most observed modes and certainly the most favorably excited modes in these stars. As long as the rotation and magnetic axes are not aligned, we can show (Bigot \& Dziembowski, 2001) that these modes displace the stellar surface along an ellipse within a plane inclined to both rotation and magnetic axes. The inclination of the plane depends mostly on the relative importance of the magnetic shift of the frequency compared to the centrifugal one. Indeed, the Coriolis shift of frequency is much smaller in roAp stars than the latter. However, the role of the Coriolis force, unlike the Lorentz and centrifugal forces, is to introduce asymmetry in the problem, so that prograde and retrograde components of the mode are affected in opposite ways. In absence of this force, the problem has a mirror symmetry in which the two non-axisymmetric components of the mode, $+m$ and $-m$, behave in the same way. Because of the asymmetry induced by the Coriolis force, these two components do not compensate, which leads to an elliptical motion of the displacement vector. In Fig. 1 we plot the mode parameters as function of the angle $\beta$ between rotation and magnetic axes, and for three different magnetic field regimes characterized by the parameter $\mu$, which is the ratio between magnetic frequency separation to centrifugal shift of frequenct The mode parameters are the dimensionless frequency, $\sigma$, the inclination of the mode plane, $\delta$, and the ellipticity angle $\psi$ whose tangent is defined as the ratio between the major and semi axes of the ellipse. The angle $\delta$ is the angle between the normal of the mode plane with the rotation axis.

We distinguish clearly two different regimes. When the rotation effects dominate $(|\mu|<1)$, the three possible dipole mode solutions of Eq. (2) are 

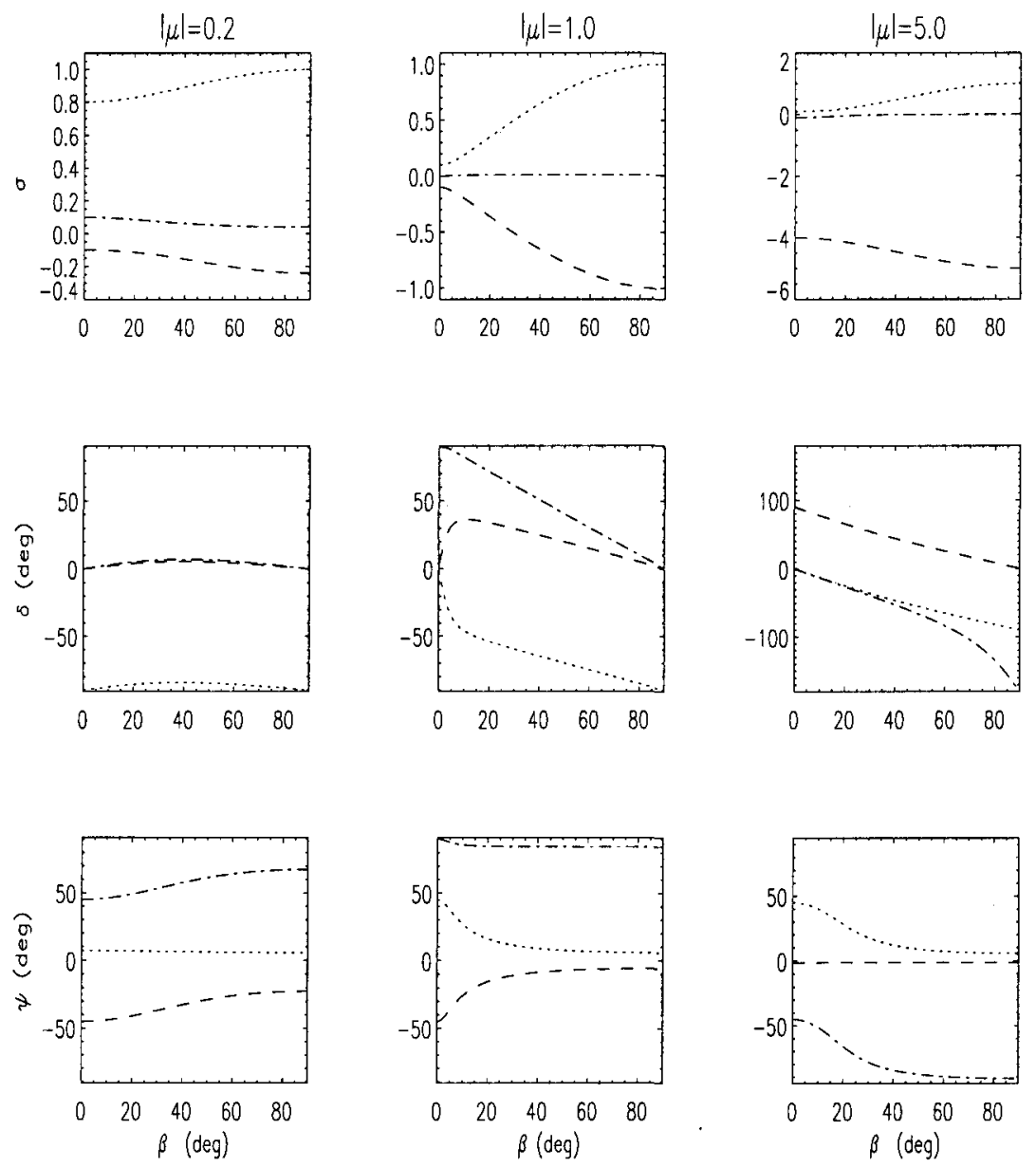

Figure 1. The fundamental parameters of dipole modes. The dimensionless frequency, $\sigma$, the inclination of the mode plane, $\delta$, and the mode ellipticity, $\psi$, are plotted on the three rows of the graph as function of $\beta$, the angle between rotation and magnetic axes. A linear polarization is defined by $\psi=0$ whereas a circular polarization is characterized by $\psi= \pm \pi / 4$. We note that an alignment with the magnetic axis requires $(|\delta|=\pi / 2-\beta, \psi=0)$ and an alignment with the rotation axis requires $(|\delta|=\pi / 2, \psi=0)$. 
either aligned with the rotation axis (with a linear polarization) or orthogonal to this axis with quasi-circular polarizations. In turn, when the magnetic field dominates $(|\mu|>1)$, one mode is linearly polarized along the magnetic axis, and two modes are orthogonal to this one. They are almost circularly polarized when the rotation and magnetic axes are aligned $\beta \approx 0$, but they tend to be linearly polarized along to orthogonal axes as $\beta \rightarrow \pi / 2$. This is a consequence of the fact that the Coriolis force, which is responsible for this ellipticity, vanishes when the rotation axis is within the mode plane, $\beta=\pi / 2$.

\section{Conclusion}

In this work we improve the treatment of roAp star pulsations in two different respects. We treat the effects of the magnetic field in outer layers of the stars with a non-perturbative theory that is needed because of the dominant magnetic pressure over the gas pressure in the outer layers of the star. We also take into account the effects of the centrifugal distortion of the star which is very significant in roAp stars, since it can be comparable to the magnetic shifts of frequencies and is much larger than the Coriolis shift. We also consider the case of dipole mode pulsations which are crucial to interpret data of roAp stars since they are the most observed modes. We show that the displacement vectors associated with these modes displace the stellar surface along an ellipse whose orientation in general is between magnetic and rotation axes.

The conclusion is that the rotation effects are sufficiently strong in roAp stars so that the mode orientation in general really differs from alignment with the magnetic axis, as it is generally assumed.

\section{References}

Bigot, L., Provost, J., Berthomieu, G., Dziembowski, W. A., \& Goode, P.R. 2000, A\&A, 356, 218

Bigot, L. \& Dziembowski, W.A., 2001, A\&A, submitted

Cunha, M. S. \& Gough, D.O. 2000, MNRAS, 319, 1020

Dziembowski, W.A. \& Goode, P.R. 1985, ApJ, 296, L27

Dziemboski, W.A. \& Goode, P.R. 1996, ApJ, 458, 338

Kurtz, D.W. 1982, MNRAS, 200, 807 